\title{
Engaging older adults in the visualization of sensor data facilitated by an open platform for connected devices
}

\author{
Christian Bock ${ }^{\mathrm{a}, \mathrm{d}}$, George Demiris ${ }^{\mathrm{a}, \mathrm{c}, *}$, Yong Choi ${ }^{\mathrm{a}}$, Thai Le ${ }^{\mathrm{a}}$, Hilaire J. Thompson ${ }^{\mathrm{c}}$, \\ Arjmand Samuel ${ }^{\mathrm{b}}$ and Danny Huang ${ }^{\mathrm{b}}$ \\ ${ }^{a}$ Biomedical Informatics and Medical Education, School of Medicine, University of Washington, \\ Seattle, WA, USA \\ ${ }^{\mathrm{b}}$ Microsoft Research, Seattle, WA, USA \\ ${ }^{\mathrm{c}}$ Biobehavioral Nursing and Health Systems, School of Nursing, University of Washington, Seattle, WA, \\ USA \\ ${ }^{\mathrm{d}}$ Ruprecht-Karls-University of Heidelberg, Heidelberg, Germany
}

Received 22 January 2016

Accepted 12 February 2016

\begin{abstract}
.
BACKGROUND: The use of smart home sensor systems is growing primarily due to the appeal of unobtrusively monitoring older adult health and wellness. However, integrating large-scale sensor systems within residential settings can be challenging when deployment takes place across multiple environments, requiring customization of applications, connection across various devices and effective visualization of complex longitudinal data.

OBJECTIVE: The objective of the study was to demonstrate the implementation of a smart home system using an open, extensible platform in a real-world setting and develop an application to visualize data real time.

METHODS: We deployed the open source Lab of Things platform in a house of 11 residents as a demonstration of feasibility over the course of 3 months. The system consisted of Aeon Labs Z-wave Door/Window sensors and an Aeon Labs Multi-sensor that collected data on motion, temperature, luminosity, and humidity. We applied a Rapid Iterative Testing and Evaluation approach towards designing a visualization interface engaging gerontological experts. We then conducted a survey with 19 older adult and caregiver stakeholders to inform further design revisions.

RESULTS: Our initial visualization mockups consisted of a bar chart representing activity level over time. Family members felt comfortable using the application. Older adults however, indicated it would be difficult to learn to use the application, and had trouble identifying utility. A key for older adults was ensuring that the data collected could be utilized by their family members, physicians, or caregivers.

CONCLUSIONS: The approach described in this work is generalizable towards future smart home deployments and can be a valuable guide for researchers to scale a study across multiple homes and connected devices, and to create personalized interfaces for end users.
\end{abstract}

Keywords: Data display, remote sensing technology, aged

\footnotetext{
${ }^{*}$ Corresponding author: George Demiris, University of Washington, BNHS Box 357266, Seattle, WA 98195, USA. Tel.: +1 206221 3866; E-mail: gdemiris@uw.edu.
}

0928-7329/16/\$35.00 (c) 2016 - IOS Press and the authors. All rights reserved

This article is published online with Open Access and distributed under the terms of the Creative Commons Attribution NonCommercial License (CC BY-NC 4.0). 


\section{Introduction}

In the field of gerontology, the use of smart home sensor systems is growing primarily due to the appeal of unobtrusively monitoring older adult health and wellness. However, the deployment of sensor systems has often been limited to academic or laboratory settings. This is due to the challenges of managing multiple simultaneous installations, integrating different types of sensors, and disseminating meaningful information back to consumers. In this paper we describe a project aimed at addressing many of these challenges.

We report the deployment of commercially available sensors within a multi-person residence using the Lab of Things platform for connected devices [1,2]. Lab of Things is a software platform that reduces the burden of deploying sensor systems across different environments while also serving as a central hub to manage connected devices, store data and analyze data. Successful deployment is only part of the challenge; processing and displaying information in a way that is easy to understand and process for stakeholders is key to the success of such a system. We address this challenge through a use case in which data collected from a smart home were integrated into a real time visualization application which was then evaluated with older adults and caregivers. This work demonstrates a holistic workflow for sensor deployment that feeds back to consumers by allowing them to access the data in an effective and efficient manner.

\subsection{Smart home sensor systems for older adults}

As defined by Demiris and colleagues, smart homes are residences that integrate technology within the home to enhance residents' safety and monitor health and wellness [3]. Smart home sensor technologies have a unique opportunity to support successful aging of the growing older adult population by identifying potential patterns in health, detecting anomalous activities, and increasing quality of life [3]. Existing research has shown that older adults are motivated to receive data from smart homes that provide insight into their health status [4] and that sensor systems in their homes have the potential to enhance their lives [5]. Furthermore, Alam et al. classify elements of smart homes into three functional groups: comfort, healthcare and security [6]. Smart home devices cover a broad spectrum including pressure sensors, cameras, contact switches, motion sensors and other electronic devices integrated within the home.

\subsection{Lab of Things as a deployment platform}

A key burden to setting up smart home sensor systems is customizing deployment based on differences across homes and sensor devices. In order to alleviate the technical challenges of deployment, we leveraged the open source Lab of Things software platform developed by Microsoft Research [1,2]. This allowed us to focus on analysis of sensor data and development of a real time visualization application. Lab of Things is a shared infrastructure simplifying not only installation of sensors in multiple home environments, but also development of smart-home applications for processing sensor data. The Lab of Things consists of a HomeOS system installed on a PC for each smart home setting. This is termed a HomeHub. Sensors within the home connect to the HomeHub through drivers and their own protocols for network transmission. The HomeHub connects to the cloud-side component of Lab of Things for data storage and central management. This allows Lab of Things to monitor, update, and remote access deployment systems across multiple environments [1,2]. The Lab of Things is in early use across various academic and applied projects [7]. It is an open source platform available to anyone interested in registering to use it. 


\subsection{Visualization approaches for sensor data}

Though Lab of Things manages smart home system deployment and integration, a gap still exists in how to transform the data created into relevant information for consumers, in our case, older adults and caregivers. One approach to this is through visualizations that synthesize the dense nature of sensor data and present them in a way that facilitates insights. The literature on visualization of sensor data is limited to a handful of studies. Wang and Skubic visualized motion data from a smart home through density maps $[8,9]$. The number of motion sensor hits was recorded and aggregated along a 2D grid representing hours within the day by days within the month. O'Brien et al. used a similar grid visualization approach, however the grid was spatially overlaid on a floor map [10]. This provided spatial information on motion sensor activity from room to room. Le and colleagues used streamgraphs and radial plots to visualize longitudinal motion sensor activity while maintaining spatial location [11]. Juarez et al. presented an alternative approach to visualizing activities in different locations of the house by using a model of multiple temporal axes [12]. Though the preceding literature provides insights into different approaches towards visualizing sensor data, they often presented the visualizations with a focus on clinical assessment. Given that older adult information needs differ from those of health care providers [13], visualization designs should be considered carefully to reflect these differences. Developing appropriate visualizations for older adults provides a valuable resource to increase access to knowledge for older adults and empower them to become more involved within their own care.

\subsection{Integrating environmental sensor data}

The prior visualization work focuses on analyzing motion sensor data to infer different levels of behavior. One key difference in our work is the integration of environmental sensor data to that of motion. Environmental data within our study included temperature, light, and humidity; all factors that could contribute to comfort within an adult's living residence. This is an added level of information that complements motion sensor data; though it also presents a visualization challenging in representing potential relationships amongst the data. Combining behavioral sensing data (e.g. mobility, sleep) with environmental parameters such as light or humidity allows for an examination of well-being of residents in a specific context and potentially the identification of parameters that contribute to or even explain behavioral observations (e.g., a resident who displays a decline in sleep quality when the room temperature increases or experiences restlessness at night when humidity decreases). This contextual examination of behavioral sensing helps tailor and control environmental aspects of a residential infrastructure to improve well-being. Thus, smart home sensor data can reveal medical emergencies (e.g., a fall that is manifested by lack of motion sensor firings) but also ways to improve well-being unrelated to an adverse event (e.g., improving temperature or lighting settings to maximize comfort and well-being).

\section{Materials and methods}

\subsection{Visualization needs and design guidelines}

We reviewed existing literature on design guidelines for older adults. This included guidelines both generalized and for sensor applications. Work by Le et al. highlighted the value older adults place in viewing longitudinal trends of data, in particular to identify gradual differences in health [11]. Further 
Table 1

Visualization design guidelines

\begin{tabular}{ll}
\hline General design component & Heuristics \\
\hline Interaction design: designing the way users work with & - Make obvious what is clickable \\
websites & - Make clickable items easy to target and hit \\
& - Minimize vertical scrolling; eliminate horizontal scrolling \\
Visual design: designing the pages & - Make pages easy to skim or scan \\
& - Make elements on the page easy to read \\
& - Visually group related topics \\
Information design: writing and formatting the content & - Use adequate white space \\
\hline
\end{tabular}

work by Mynatt et al. identified the role of smart homes in identifying crisis situations, providing everyday cognitive support, and raising awareness of daily life and long-term trends [14]. Based on this research in the literature, our primary aims with the design were to: 1) create a simple and familiar visualization of longitudinal activity, 2) support exploration across spatial locations within the smart home, and 3) monitor potential correlations in physical environment within the home. Chisnell et al. provided a set of heuristics for designing web sites for older adults [15]. Similarly, the National Institute on Aging also provided a set of guidelines for designing older adult appropriate websites. Though not all of the guidelines fit within the context of data visualization, a subset of these are described in Table 1.

\subsection{Sensor and system deployment}

We deployed the Lab of Things platform in a house of 11 residents as a demonstration of feasibility over the course of 3 months. The system consisted of one multi-sensor and two door/window sensors. These sensors were commercially available and consisted of two Aeon Labs Z-wave Door/Window sensors and an Aeon Labs Multi-sensor. The door/window sensor recorded a binary on/off signal when the magnetic switch was activated while the multi-sensor collected data on motion, temperature, luminosity, and humidity. The door/window sensors were installed at the front entrance of the residence and on the refrigerator door. The multi-sensor was installed in the kitchen above the kitchen island. The multisensor was mounted 3 meters above the ground resulting in an ellipsis effective range with a transverse diameter of 6 meters and a conjugate diameter of 10 meters. The Lab of Things (LoT) platform was deployed on laptop located on a shelf in the kitchen to be as unobtrusive as possible. The set-up had relatively low cost hardware with entire cost of the sensors and the laptop approximately at $\$ 500$ (with door/window sensors at approx. \$40 each, the multisensory at approx. \$50 and the netbook ad Z-wave USB stick at approx. $\$ 300$ at the time). The sensor devices use multiple layers of security, from device codes to signal encryption, to avoid security breaches and interference. They all utilize Gen5's AES-128 data encryption. The generated data include sensor firings (e.g., when a door is opened) or environmental parameters such as temperature and humidity (in the case of the multi-sensor). Thus, the sensor data stored in the cloud do not include any personal or identifiable data. The participants' personal data (such as demographics) were stored locally and not on the cloud, in a separate secure, encrypted, HIPAA compliant database and linked to sensor data only through a unique participant identifier number.

\subsection{Design process}

We applied a Rapid Iterative Testing and Evaluation (RITE) approach towards designing the visualizations [16]. This involved two rounds of iterative prototyping with gerontological experts. We presented 


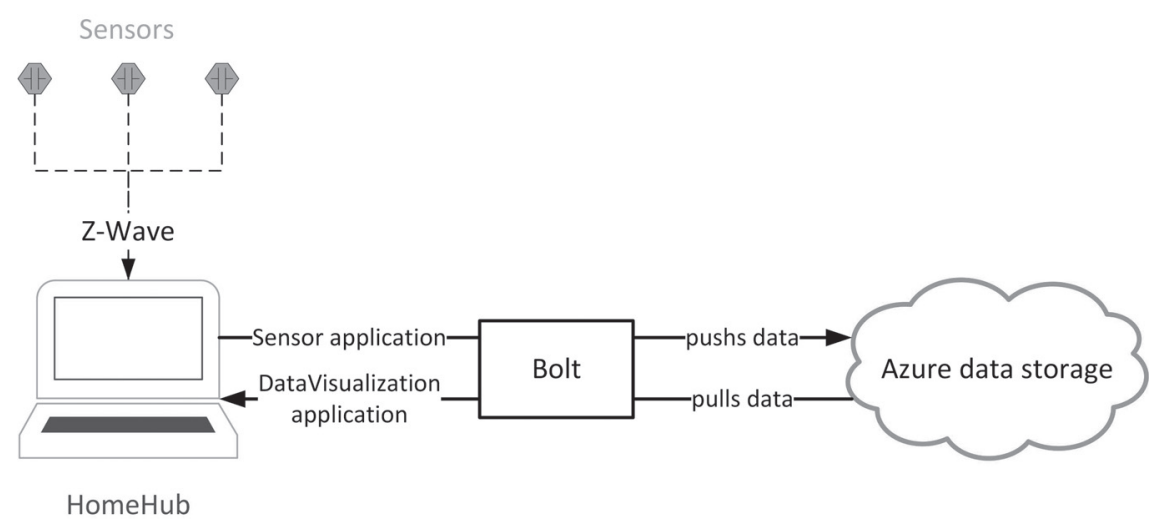

Fig. 1. System architecture diagram. Sensors transmit data to the HomeHub via the Z-wave transmission protocol. The HomeHub sends data to and retrieves data from cloud storage using the Bolt API.

early mockups to a group of 10 gerontology experts to gather feedback and design guidelines. This was used to iterate on the design across two cycles. We developed the visualization application based on the design guidelines and domain expert feedback. We then conducted a third evaluation through a survey with older adult and caregiver stakeholders to inform further revisions to the designs.

\subsection{Application development}

We developed the application through a combination of HTML, CSS, and JavaScript for the client side. We used a service-oriented architecture to communicate with the server machine, represented by the HomeHub on the Lab of Things platform. All server code was implemented using C\#. We used the Bolt API to both push and pull data to and from the Microsoft Azure Cloud [17]. The sensors push data into the cloud storage, which are then pulled by the data visualization application to generate the graphs (Fig. 1).

\subsection{Application architecture}

The classical client-server model is extended through a second communication channel between the HomeHub and Microsoft's Azure Data Storage. Figure 2 illustrates how a request from a web browser (client) - triggered by interacting with the application - is interpreted and forwarded by the HomeHub to the Database as aforementioned. The results of the database queries are processed by the HomeHub itself and a csv like string is created to be consumed by the JavaScript code in the front end. In this component of the system, the appearance of the graph is defined and plotted in the web browser using the dygraph.js library.

\section{Results}

\subsection{Sensor visualization applications}

Our initial mock-ups consisted of a bar chart representing activity level over time. A "Norm Activity Index" was provided as a reference for average activity level. We used color as an encoding for activity 


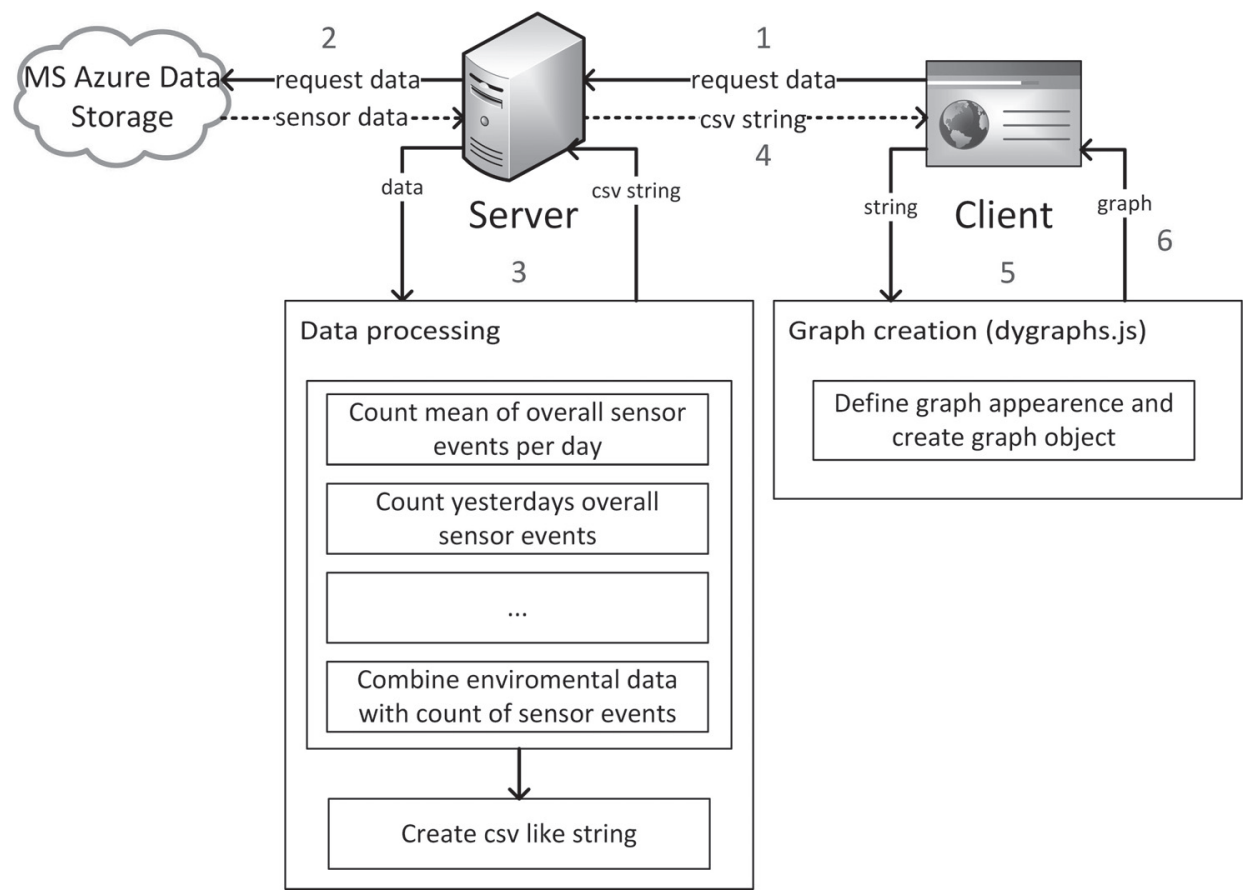

Fig. 2. 1: The client application requests data from the HomeHub by opening and interacting with the application. 2: the HomeHub queries requested data from the MS Azure Data Storage. 3: Received data gets processed by the HomeHub and required numbers are calculated. A consumable csv like string is created and sent to the client side (4). 5: The string gets processed and fed into the dygraphs.js charting library which results in the visualization of the graph (6).

level relative to the norm in the bar chart. Blue indicated activity above the norm, red indicated activity below the norm, and black indicated activity at approximately the norm. Below the bar chart was a dual axis line graph providing information on both temperature and humidity. We included these environmental variables on the same line graph to show the close correlation between the two factors. After an initial heuristic evaluation with gerontological experts applying guidelines by Chisnell et al. [15], we reiterated on the designs. Repeating a similar process, we reviewed the second design with experts and iterated based on feedback from the heuristic evaluation.

The application pulls live sensor data from the Microsoft Azure cloud storage and uses it to generate graphs of sensor activity level and environmental measures. In real time, consumers are able to view sensor events day-to-day relative to an average event level, which is represented by the mean of the number of sensor events of the last fourteen days. End users are able to switch between spatial locations within the deployment environment and also to view environmental measures collected from the sensors. This includes humidity, temperature, and luminosity. The total sensor events are also displayed on the environmental graphs, allowing users to correlate the two data sources. In our example, there was a close association between temperature and sensor activity, consistent with a hypothesis that increased sensor activity is related to more people within an environment. Within a kitchen environment, in addition to more people being present, activities such as using the stove or oven could contribute to the increased temperature. We also noticed a close relationship between luminosity and activity level, with luminosity levels increasing slightly before sensor activity. The pattern corresponds to a diurnal cycle of activity and light. 

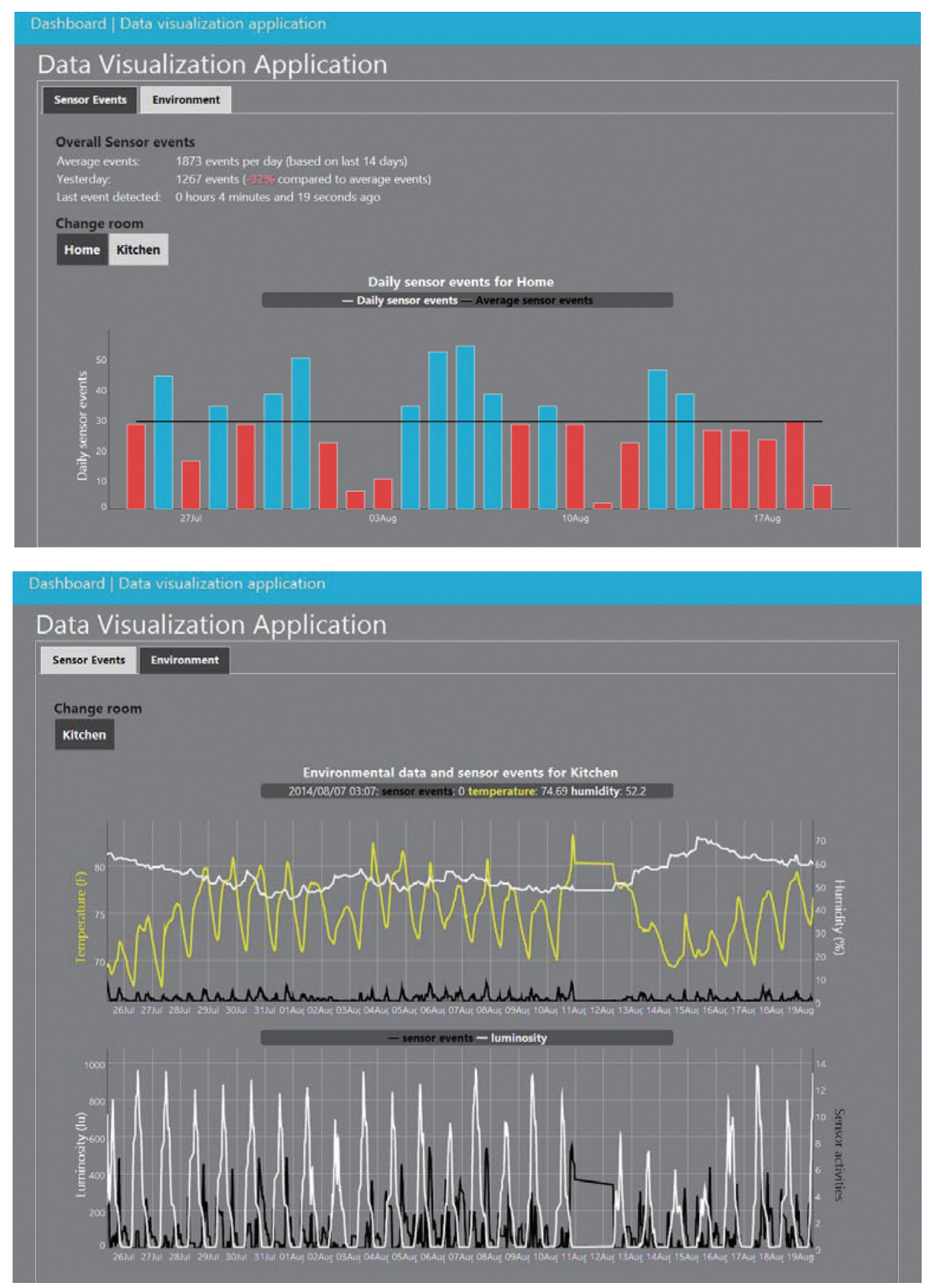

Fig. 3. Final application developed incorporating live sensor data from the pilot study. The application pulls sensor data from cloud storage and uses the dygraphs.js library to generate visualizations. The dashboard separates sensor events from environmental data across different tabs.

\subsection{Evaluation with stakeholders}

Nineteen participants completed the online survey. Of those 12 participants were female, and 7 were male. There were 7 older adult participants at least 60 years old while the remaining were family members/caregivers. The most important information for stakeholders was knowing when the last sensor event was detected (8/12 family members/caregivers and 3/7 older adults indicated it was important to very important). This was contrasted with showing average events or comparison of sensor events to an average which both stakeholder groups rated low on importance. Participants indicated that it was impor- 
tant to be able to switch activity views amongst rooms and also to show a total activity level house-wide, consistent with features available within the application. However a participant also noted as open-ended feedback the value of showing the amount of time spent in each room as opposed to solely the number of sensor events.

When examining responses to the SUS questionnaire, family members/caregivers felt comfortable using the application indicating that they could quickly learn to use the application and would not need the support of a technical person. As one family member/caregiver noted, this struck a good balance of providing relevant information in a simple manner without being overly obtrusive, capturing basic sensor events and environmental data as "good indicator of activity and health without providing too personal of information."

Older adults however indicated it would be difficult to learn, and had trouble identifying utility with the application. A key for older adults was providing information on how the data collected could be utilized by their family members, physicians, or caregivers. An older adult highlighted this issue of utility, stating that he had not thought of the application as useful at first, but upon discussing with his daughter he realized that there was value in cases where "the family member or caregiver could be alerted if there hadn't been any sensor events for a predetermined length of time."

\section{Discussion}

Being able to implement smart homes efficiently through an open and extensible platform is of great benefit from a re-search perspective. We experimented with the Lab of Things platform as a resource to facilitate deployment without worrying about technical device details. We found that the platform did successfully accommodate different sensor devices and that set up allowed a more central source of control for managing the smart home system. Community developed drivers allowed us to connect with most common sensor devices. From a development point of view, a weakness of the system was that the Bolt framework only allowed very simple queries on the data saved in cloud storage. Especially for a data visualization application, this led to heavy data processing on the server side, producing latency of performance. Furthermore we noticed that for future applications, it would be of advantage to have a generic, out of the box solution for data visualization provided by the Lab of Things platform.

The evaluation of our visualization application with stakeholders highlighted significant differences between how family members/caregivers and older adults perceived the application. Though family members/caregivers found the application easy to learn and identified value in having the information available to monitor older adult wellness, older adults did not share this same view. Instead, older adults found the visualization complex and difficult to learn, partially confounded by an unclear sense of how the information could be used. This lack of perceived usability and utility can be a significant barrier to engagement [19]. Simplifying the visualization would help reduce usability issues, however it is also important to demonstrate, through use case examples, how the information could be helpful. This would alleviate the trade-offs older adults must make between privacy and utility when deciding to have sensors within the home.

Our work demonstrates a step towards making smart home information more accessible to residents. The flexibility of Lab of Things to control and manage smart home systems allows customization by the resident. Creating user friendly visualizations that effectively represent complex and often dense sensor data allows older adults to access the information in an effective manner, empowering their engagement in care and health monitoring. 
Study limitations include a small sample size in one site only with a limited follow-up period. Given its emphasis on feasibility and acceptance, this study did not address challenges such as interoperability of the interfaces with other systems such as electronic medical records nor the impact of sensor data visualizations on actual clinical decision making.

\section{Conclusion}

The current growth of the older adult population, rising health care costs, and the desire to maintain independence are all motivating factors in smart home research. Older adults have expressed interest in smart home technologies [5]; one way to facilitate their adoption is through visualizations that incorporate data from smart home sensors into relevant and insightful resources. A challenge with many systems is the customization required to deploy smart homes across various environments and sensors. We demonstrated the implementation of a smart home system using an open, extensible platform. In addition, we closed the loop between the research and applied nature of smart homes by developing an application to visualize data real time. This was evaluated with older adults and family members/caregivers as direct stakeholders of the information. The approach described in this work is generalizable towards future smart home deployments and can be a valuable guide for researchers to scale a study across multiple homes and connected devices.

\section{References}

[1] Brush AJB, Jung J, Mahajan R, Scott J. HomeLab: Shared Infrastructure for Home Technology Field Studies. Proceedings of the 2012 ACM Conference on Ubiquitous Computing. New York: ACM; 2012. p. 1108-13. doi: 10.1145/ 2370216.2370450.

[2] Brush AJB, Filippov E, Huang D, et al. Lab of Things: A Platform for Conducting Studies with Connected Devices in Multiple Homes. Proceedings of the 2013 ACM Conference on Pervasive and Ubiquitous Computing Adjunct Publication. New York: ACM; 2013. p. 35-8. doi: 10.1145/2494091.2502068.

[3] Demiris G, Hensel BK. Technologies for an aging society: A systematic review of "smart home" applications. Yearb Med Inform. 2008; 3: 33-40.

[4] Lee ML, Dey AK. Embedded assessment of aging adults: A concept validation with stakeholders. InPervasive Computing Technologies for Healthcare (PervasiveHealth), 2010 4th International Conference on-NO PERMISSIONS 2010. IEEE p. 1-8. doi: 10.4108/ICST.PERVASIVEHEALTH2010.8849.

[5] Demiris G, Rantz MJ, Aud MA, Marek KD, Tyrer HW, Skubic M, Hussam AA. Older adults' attitudes towards and perceptions of "smart home" technologies: A pilot study. Informatics for Health and Social Care. 2004 Jun; 29(2): 87-94. doi: 10.1080/14639230410001684387.

[6] Alam MR, Reaz MB, Ali MA. A review of smart homes - Past, present, and future. Systems, Man, and Cybernetics, Part C: Applications and Reviews, IEEE Transactions on. 2012 Nov; 42(6): 1190-203.

[7] Microsoft Research [homepage on the Internet]. Research projects using the Lab of Things [cited 2015 Apr 22]. Available from: http://www.lab-of-things.com/community.html.

[8] Wang S, Skubic M. Density map visualization from motion sensors for monitoring activity level. In Intelligent Environments, 2008 IET 4th International Conference. IET; 2008 Jul 21. p. 1-8.

[9] Wang S, Skubic M, Zhu Y. Activity density map visualization and dissimilarity comparison for eldercare monitoring. Information Technology in Biomedicine, IEEE Transactions on. 2012 Jul; 16(4): 607-14. doi: 10.1109/TITB.2012. 2196439.

[10] O’Brien A, McDaid K, Loane J, Doyle J, O’Mullane B. Visualisation of movement of older adults within their homes based on PIR sensor data. In Pervasive computing technologies for healthcare (PervasiveHealth), 2012 6th international conference. IEEE; 2012 May 21. p. 252-259.

[11] Le T, Reeder B, Chung J, Thompson H, Demiris G. Design of smart home sensor visualizations for older adults. Technology and health care: Official journal of the European Society for Engineering and Medicine. 2014 Jan; 22(4): 657-66. doi: 10.3233/THC-140839. 
[12] Juarez JM, Ochotorena JM, Campos M, Combi C. Multiple temporal axes for visualising the behaviour of elders living alone. InHealthcare Informatics (ICHI), 2013 IEEE International Conference. IEEE; 2013 Sep 9. p. 387-395.

[13] Reeder B, Le T, Thompson HJ, Demiris G. Comparing information needs of health care providers and older adults: Findings from a wellness study. Studies in health technology and informatics. 2013; 192: 18-22.

[14] Mynatt ED, Rowan J, Craighill S, Jacobs A. Digital family portraits: supporting peace of mind for extended family members. InProceedings of the SIGCHI conference on Human factors in computing systems. New York: ACM; 2001 Mar 1. p. 333-340. doi: 10.1145/365024.365126.

[15] Chisnell DE, Redish JC, Lee AM. New heuristics for understanding older adults as web users. Technical Communication. 2006 Feb 1; 53(1): 39-59.

[16] Medlock MC, Wixon D, Terrano M, Romero R, Fulton B. Using the RITE method to improve products: A definition and a case study. Usability Professionals Association. 2002 Jul 8; 51.

[17] Gupta T, Singh RP, Mahajan AP. Bolt: Data management for connected homes. In Proceedings of the 11th USENIX Symposium on Networked Systems Design and Implementation (NSDI 14). 2014 Apr 2. p. 243-256.

[18] Brooke J. SUS: A “quick and dirty” usability scale. Usability Evaluation in Industry. London: Taylor \& Francis; 1996 p. 189-194.

[19] Flavián C, Guinalíu M, Gurrea R. The role played by perceived usability, satisfaction and consumer trust on website loyalty. Information \& Management. 2006 Jan; 43(1): 1-14. doi: 10.1016/j.im.2005.01.002. 